Supplementary Information

PLGA Nanoparticles Co-Delivering siRNAs against Programmed Cell Death Protein-1 and Its Ligand Gene for Suppression of Colon Tumor Growth

Seo Young Kwak, Seonmin Lee, Hee Dong Han, Suhwan Chang, Kyu-pyo Kim, Hyung Jun Ahn 


\section{S1. Materials}

DNA primers and siRNA oligoes against mouse PD-1 and PD-L1 gene, scrambled siRNA (scRNA), and Cy5.5-labeled siRNA were purchased from Bioneer Co. (Daejon, Korea), of which sequences are shown in Table S1. Poly(lactic-co-glycolic acid) (PLGA), poly-L-lysine (PLL), polyvinyl alcohol (PVA), polyinosinic-polycytidylic acid sodium salt (poly(I:C)), and chloroform were purchased from Sigma-Aldrich (UK). Each primary antibodies were purchased as follows; anti-mouse PD-1 (ProSci, CA, USA), anti-mouse PD-L1 (Cell signaling, MA, USA), anti-mouse CD8a (eBioscience, CA, USA), and anti-mouse p-S6K (Abcam, USA). FITC anti-mouse CD3e, PE anti-mouse CD8a, APC anti-mouse CD4, APC anti-mouse CD8a, APC anti-mouse CD11c, FITC anti-mouse CD11c, APC anti-mouse F4/80, APC anti-mouse CD40, APC anti-mouse CD86, FITC anti-mouse PD-1, FITC anti-mouse PD-L1, and FITC anti-mouse INF- $\gamma$ antibodies were obtained from Biolegend (CA, USA). PD-L1-blocking mAb (10F.9G2) and PD-1-blocking mAb (29F.1A12) was obtained from BioLegend. The purified rat anti-mouse CD16/CD32 (mouse BD Fc block) and GolgiPlug ${ }^{\mathrm{TM}}$ was obtained from BD Biosciences (CA, USA). Recombinant murine IL-2 was purchased from PeproTech (NJ, USA). Each experimental kits were purchased as follows; CellTrace ${ }^{\mathrm{TM}} \mathrm{CFSE}$ cell proliferation kit, StepOnePlus real-time PCR system, and High Capacity cDNA Archive Kit from Thermo Fisher Scientific (MA, USA), CD8a ${ }^{+}$T Cell Isolation Kit from Miltenyi Biotec (Germany), APO-BrdUTM TUNEL assay kit from Invitrogen (USA), RNeasy Mini Kit from Qiagen (USA), Cell Counting Kit-8 cytotoxicity assay from Dojinjo Molecular Technologies (Japan), and IHC staining kit from VECTOR Laboratories (CA, USA).

\section{S2. DLS Analysis and TEM Images}

The particle size and zeta potential of siRNA@PLGA NPs and (I:C)@PLGA NPs were determined using a Malvern Nano ZS and Zeta-sizer (Malvern). All samples were serially diluted with PBS ( $\mathrm{pH} 7.4$ ), and then collected data at a scattering angle of $173^{\circ}$ with a standard 633 nm laser. The particle size and morphology of siRNA@PLGA NPs and (I:C)@PLGA NPs were visualized using a CM30 electron microscope (Philips). Samples were negatively stained with a droplet of $2 \%$ aqueous uranyl acetate and the grid was dried overnight.

\section{S3. Cell Culture}


Murine MC38 colon carcinoma cells, purchased from American Type Culture Collection (ATCC, US), were cultured in RPMI 1640 and DMEM medium respectively, which were supplemented with $10 \%$ FBS and $1 \%$ penicillin-streptomycin solution. The cells were grown in a $37{ }^{\circ} \mathrm{C}$ and $5 \% \mathrm{CO}_{2}$ incubator until reaching $60 \%$ confluency. Ovalbumin-expressing B16F10 (B16F10-OVA) cells, purchased from ATCC, were cultured in DMEM medium supplemented with $10 \%$ FBS and 1\% penicillin-streptomycin solution.

\section{S4. Cytotoxicity Study of Scrambled siRNA@PLGA NPs on MC38 Cells}

MC38 tumor cells cultured at 24-well plates $\left(1 \times 10^{7}\right.$ cells/well $)$ were transfected with scRNAs@PLGA NPs $(0.1-6.0 \mathrm{mg} / \mathrm{ml})$ or PBS in serum-free medium for $4 \mathrm{~h}$, and then washed twice. After incubated in fresh medium for additional $20 \mathrm{~h}$ or $44 \mathrm{~h}, \mathrm{CCK}-8$ solution $(10 \mu \mathrm{L})$ was poured into each well. The cells were incubated for $2 \mathrm{~h}$, and then the absorbance of samples at $450 \mathrm{~nm}$ was measured by Spectra MAX 340 Microplate reader (Molecular Device, USA).

\section{S5. Basal Expression Levels of PD-1 on various immune cells}

The isolated splenocytes from naive C57BL/6 mice were pretreated with purified rat antimouse CD16/CD32 antibodies to block non-specific binding of Fc receptor-expressing cells and then stained with a combination of FITC anti-mouse PD-1 with specific marker antibodies including APC anti-mouse CD4, APC anti-mouse CD8, APC anti-mouse CD11c, or APC antimouse $\mathrm{F} 4 / 80$. On the other hand, the freshly isolated naive $\mathrm{CD}^{+} \mathrm{T}$ cells were stimulated by the immobilized anti-CD3 and anti-CD28 antibodies for 1 day to induce PD-1 expression. The stained cells with APC anti-mouse CD8 and FITC anti-mouse PD-1 antibodies were examined with flow cytometer and PD-1 expression levels in each subset were measured for the doublepositive cell population.

\section{S6. In vivo tumor targeting of siRNA@PLGA in xenograft model}

MC38 cells $\left(1 \times 10^{6}\right.$ cells $\left./ 100 \mu \mathrm{L}\right)$ were subcutaneously inoculated into the flanks of C57BL/6 mice (6 week-old female, Orient Bio, Korea). A week after inoculation, the mice were intravenously injected with Cy5.5-siRNA $(2.0 \mathrm{mg} / \mathrm{kg})$ or Cy5.5-siRNA@PLGA $(2.0 \mathrm{mg} / \mathrm{kg}$ ) via tail vein ( $\mathrm{n}=7$ per group). We measured whole body fluorescence images $6 \mathrm{~h}$ and $24 \mathrm{~h}$ post-injection for Cy5.5 signals using IVIS spectrum and IVIS Living imaging Software (Caliper Life Science, US). Next, we sacrificed the mice to excise tumor tissues and 
major organs (heart, lung, liver, spleen, and kidney) $24 \mathrm{~h}$ post-injection. The ex vivo fluorescence images of tumors and organs were measured in a same way. 


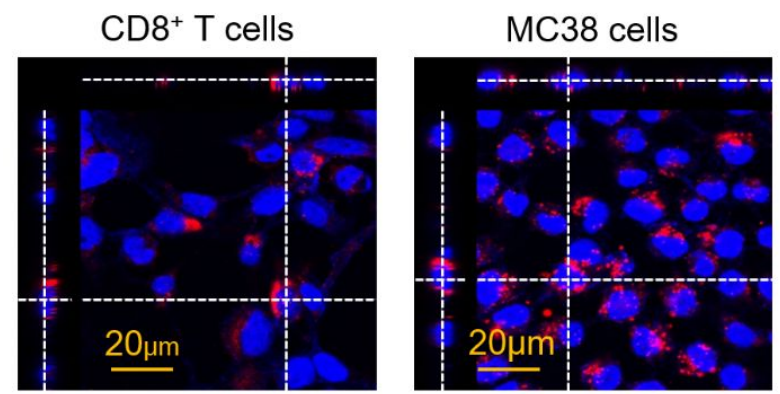

Figure S1. Cellular internalization of Cy5.5-siRNA@PLGA NPs in the CD8 ${ }^{+} \mathrm{T}$ cells and MC38 cells. Z-stack confocal microscopic images of CD8 ${ }^{+} \mathrm{T}$ cells and MC38 cells transfected with Cy5.5-siRNA@PLGA NPs in a similar manner as in the Figure 2B. Red and blue signals indicate Cy5.5-siRNA and DAPI, respectively. Scale bar: $20 \mu \mathrm{m}$. 

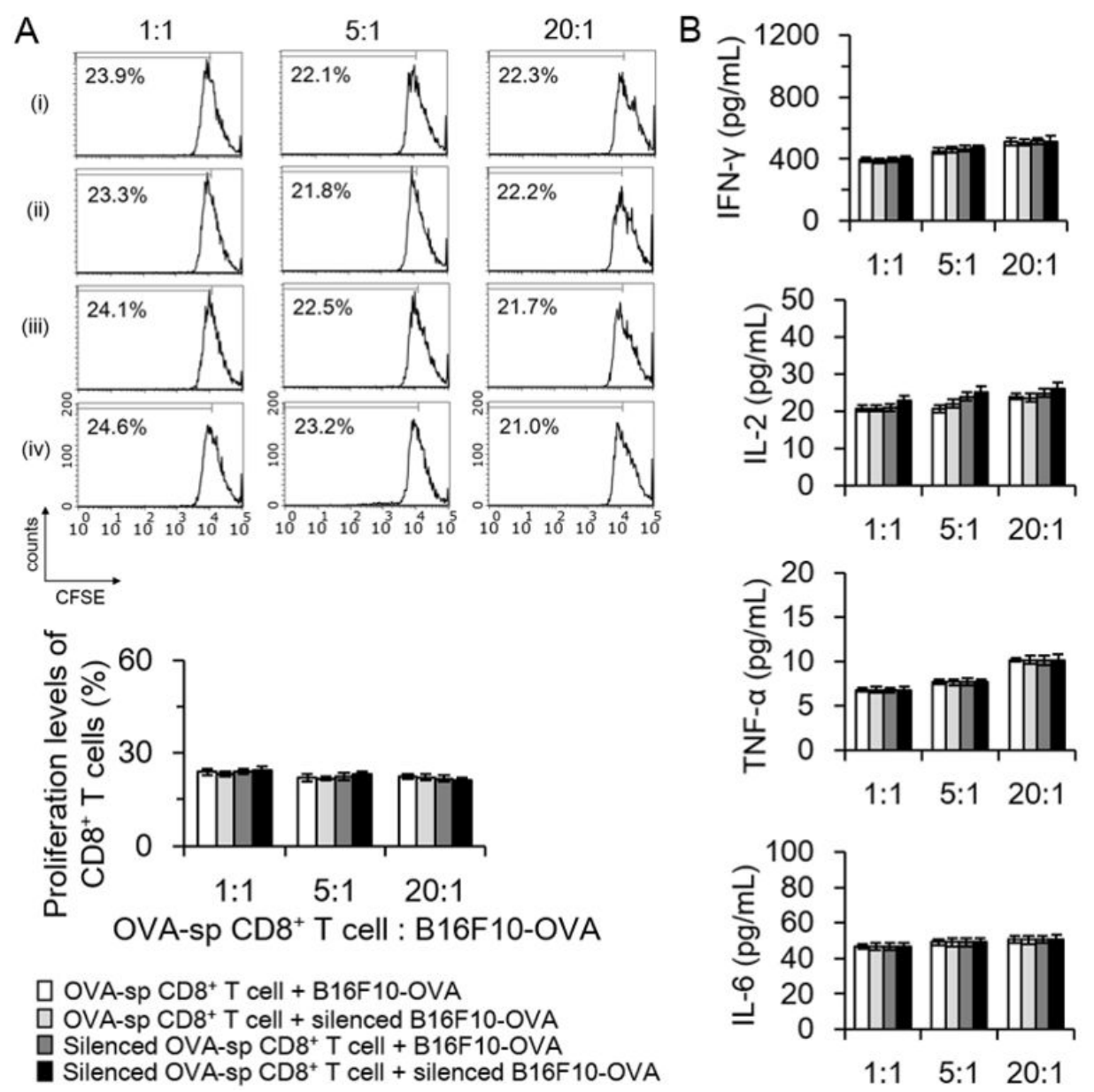

Figure $\mathrm{S} 2 . \mathrm{CD}^{+} \mathrm{T}$ cell proliferation and cytokine production upon transfection with scrambled scRNA@PLGA NPs. After B16F10-OVA cells and OVA-stimulated CD8 ${ }^{+} \mathrm{T}$ cells were transfected with scrambled scRNA@PLGA NPs or PBS, B16F10-OVA cells were co-cultured with CFSE-labeled $\mathrm{CD}^{+} \mathrm{T}$ cells at the indicated E:T ratios. Proliferative response of $\mathrm{CD} 8^{+} \mathrm{T}$ cells and production of IFN- $\gamma$, IL-2, TNF- $\alpha$, and IL- 6 were examined by flow cytometry and ELISA method, respectively. The results are presented as the mean \pm s.d. $(n=6)$. 


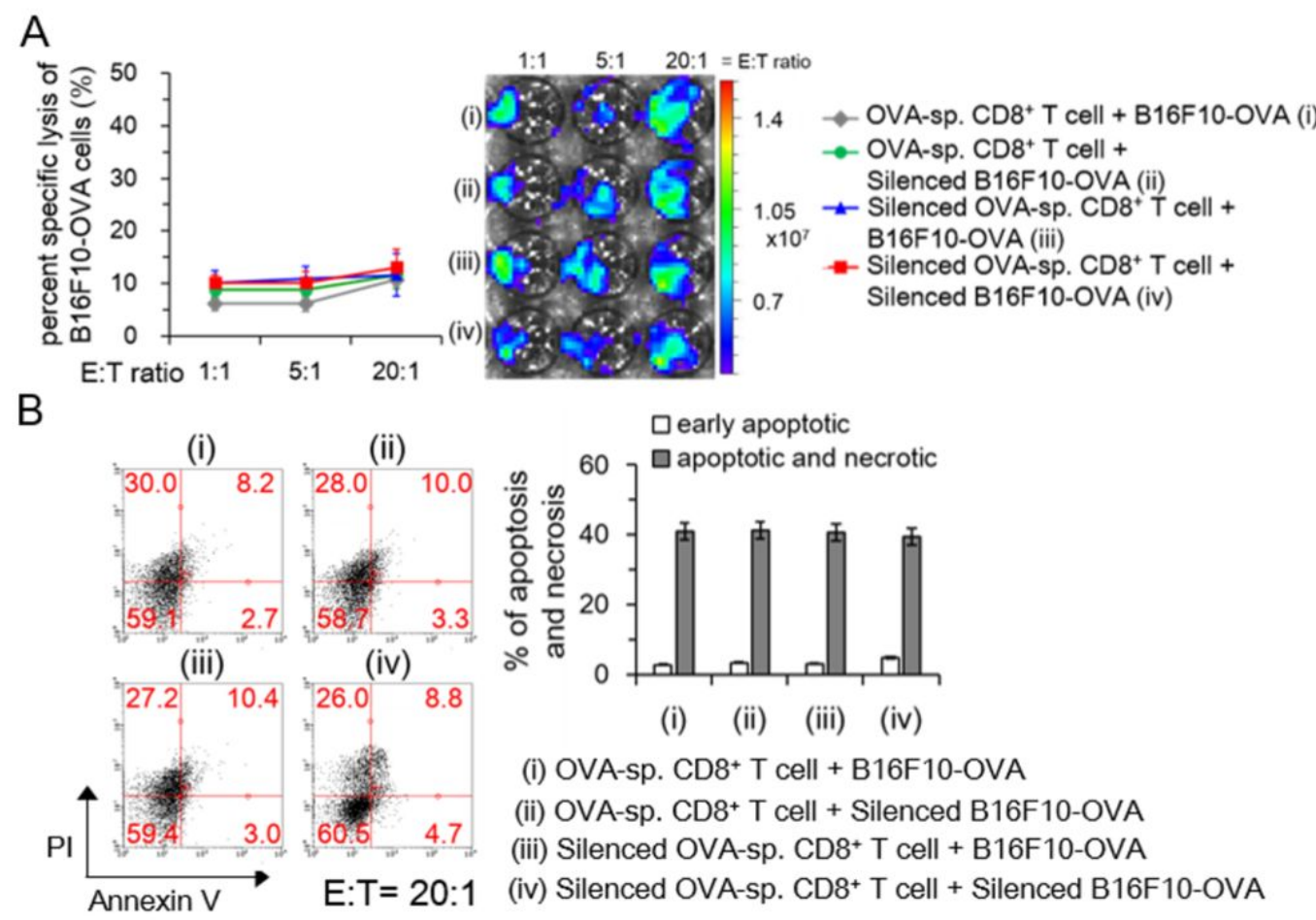

Figure S3. In vitro cytolytic activity of OVA-specific $\mathrm{CD}^{+} \mathrm{T}$ cells $(\mathrm{A})$ and induction of apoptosis in B16F10-OVA cells (B) in the co-culture system treated with scRNA@PLGA NPs. OVA-stimulated $\mathrm{CD}^{+} \mathrm{T}$ cells and B16F10-OVA cells were transfected with scrambled scRNA@PLGA NPs or PBS for $4 \mathrm{~h}$ and incubated for additional $40 \mathrm{~h}$. The results are presented as the mean \pm s.d. $(n=3)$. 


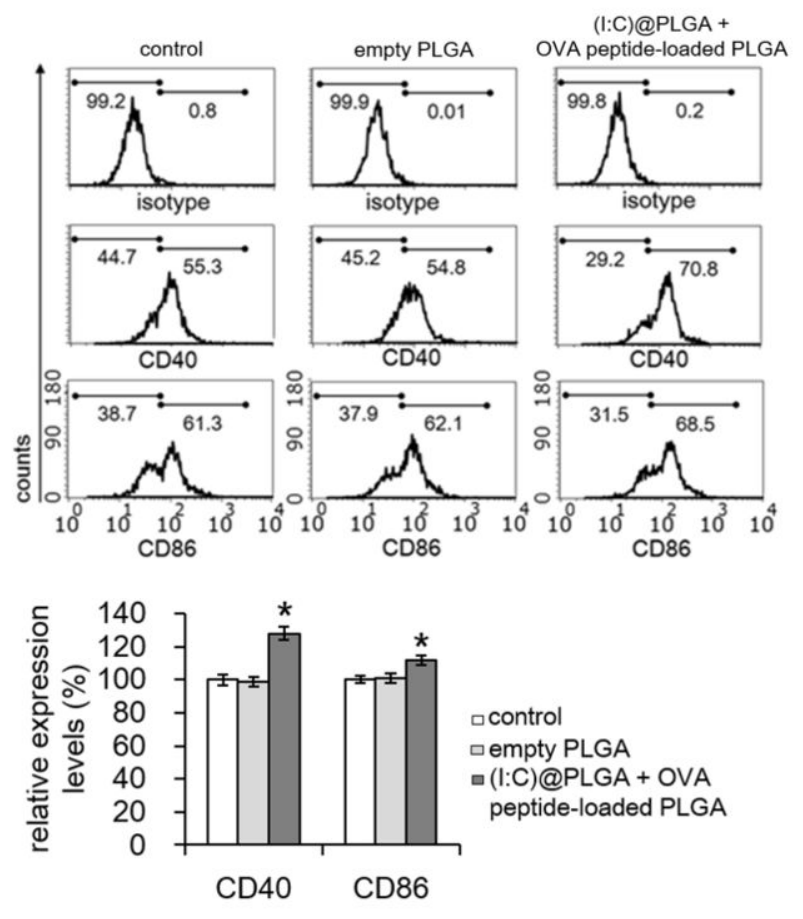

Figure S4. Maturation of DCs by (I:C)@PLGA NPs in the presence of OVA peptide. Bone marrow-derived DCs were transfected with empty PLGA NPs or (I:C)@PLGA NPs plus OVA peptide-loaded PLGA NPs, and then analyzed by flow cytometry to examine the expression of CD40 and CD86 markers on CD11 ${ }^{+}$bone marrow-derived DCs. A control sample represents the non-transfected DCs in the absence of OVA peptide. The relative expression levels of CD40 and CD86 were plotted versus those of control sample. The results are presented as the mean \pm s.d. (n=3). ${ }^{*} P<0.01$ vs. control. 


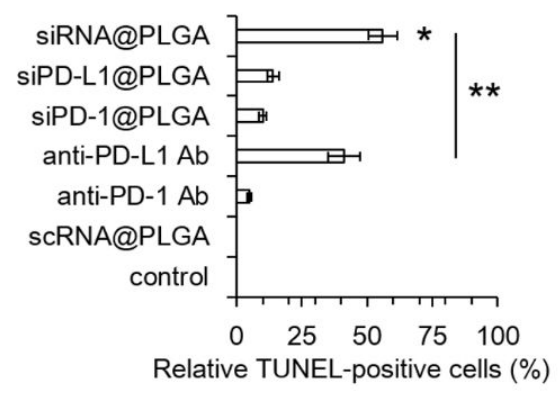

Figure S5. Apoptosis measured by TUNEL assay. TUNEL-positive cells were counted at 200x magnification. The levels of apoptosis were indicated as the percentage of TUNEL-positive cells among total cells. Five sections per tumor tissue were examined and five random fields per section were measured: control PBS group $(n=4)$, siRNA@PLGA group $(n=7)$, anti-PDL1 Ab group $(n=5)$, anti-PD-1 Ab group $(n=5)$, siPD-1@PLGA group $(n=4)$, siPDL1@PLGA group $(n=4)$, and scrambled scRNA@PLGA group $(n=4)$. The results are presented as the mean \pm s.d. $(\mathrm{n}=3) .{ }^{*} P<0.0001$ vs. control, ${ }^{* *} P>0.12$ vs. anti-PD-L1Ab group. 


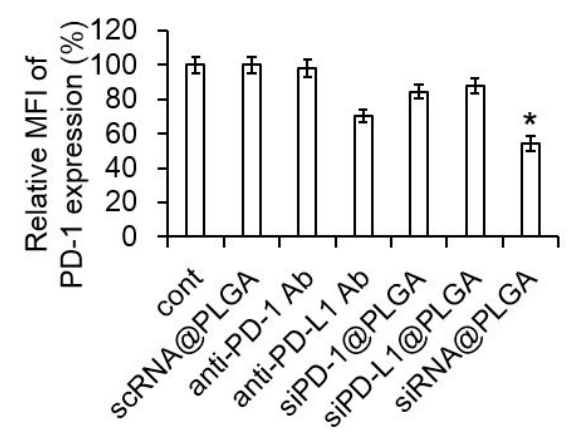

Figure S6. Reduced PD-1 expression levels in tumor-infiltrating CD8 ${ }^{+} \mathrm{T}$ cells from the treated mouse groups. The relative PD-1 expression levels in the Figure 8D are plotted versus control group. Tumor-bearing mice were randomly divided into seven groups: control PBS group ( $\mathrm{n}=$ 4), siRNA@PLGA group ( $(n=7)$, anti-PD-1 Ab group $(\mathrm{n}=5)$, anti-PD-L1 Ab group $(\mathrm{n}=5)$, siPD-1@PLGA group $(\mathrm{n}=4)$, siPD-L1@PLGA group $(\mathrm{n}=4)$, and scrambled scRNA@PLGA group $(\mathrm{n}=4)$. The results are presented as the mean \pm s.d. ${ }^{*} P<0.05$ vs. control group. 


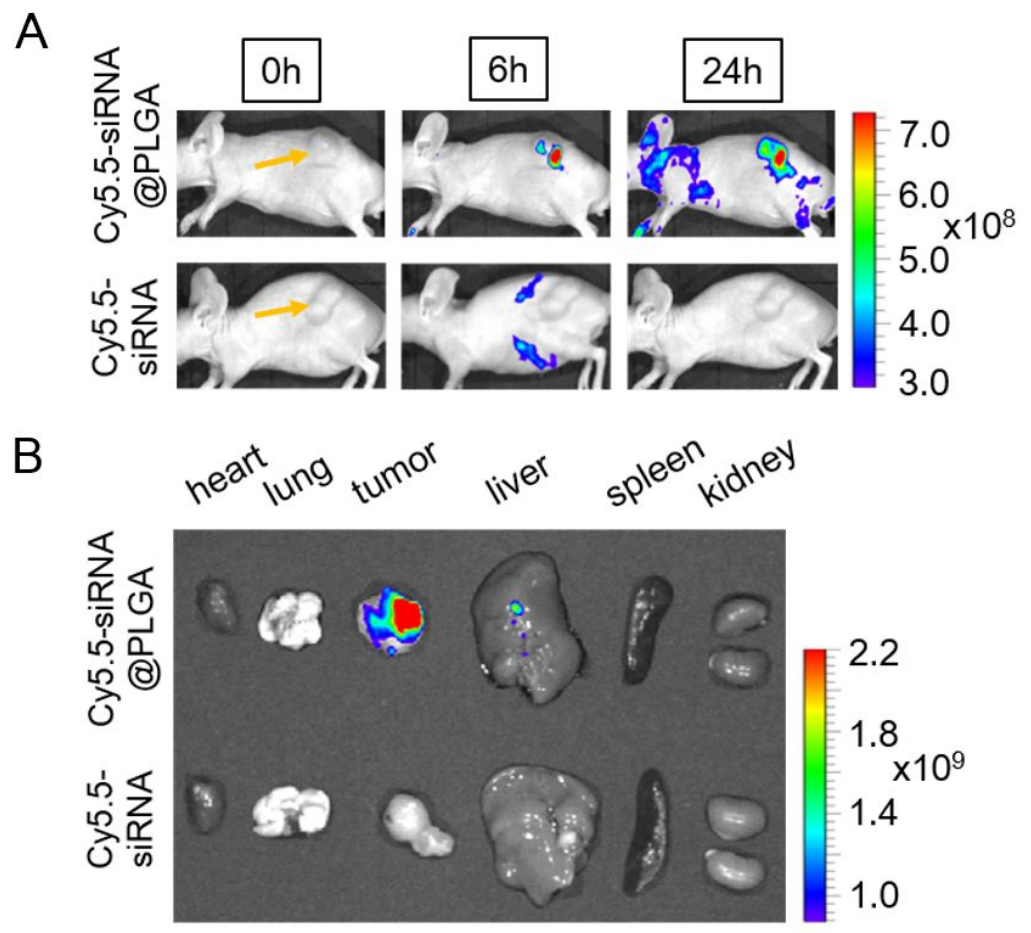

Figure S7. In vivo biodistribution and accumulation studies of siRNA@PLGA. (A) Whole body fluorescence images of intravenously injected Cy5.5-siRNA@PLGA or Cy5.5-siRNA in MC38 xenograft mice. $0 \mathrm{~h}, 6 \mathrm{~h}$, and $24 \mathrm{~h}$ after injection, each fluorescence signals of Cy5.5siRNA@PLGA or Cy5.5-siRNA were monitored in Cy5.5 fluorescence channel to track their biodistribution. Tumor sites were indicated by arrow. (B) Ex vivo fluorescence images of excised tumors and organs. The injected mice were sacrificed $24 \mathrm{~h}$ post-injection, and their tumors and major organs were excised. Each fluorescence signals of Cy5.5-siRNA@PLGA or Cy5.5-siRNA were measured in a similar manner. 


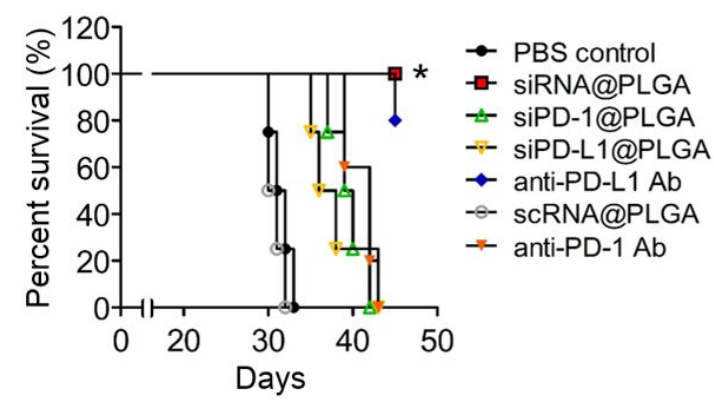

Figure S8. Mouse survival after siRNA@PLGA NPs administration. Percent survival of mice in the Figure 8A was plotted. Tumor-bearing mice were randomly divided into six groups: control PBS group $(\mathrm{n}=4)$, siRNA@PLGA group $(\mathrm{n}=7)$, anti-PD-1 Ab group $(\mathrm{n}=5)$, antiPD-L1 Ab group $(n=5)$, siPD-1@PLGA group $(n=4)$, siPD-L1@PLGA group $(n=4)$, and scrambled scRNA@PLGA group $(\mathrm{n}=4)$. ${ }^{*} P<0.001$ vs. PBS control group. 


\begin{tabular}{|l|l|}
\hline mouse PD-1 siRNA & $\begin{array}{l}5^{\prime} \text { - CACUUCUAGGGACUUGAGAdTdT-3' } \\
5^{\prime} \text {-dTdTGUGAAGAUCCCUGAACUCU-3' }\end{array}$ \\
\hline mouse PD-L1 siRNA & $\begin{array}{l}5^{\prime} \text { - GACUCAAGAUGGAACCUGAdTT-3' } \\
5^{\prime} \text {-dTdTCUGAGUUCUACCUUGGACU-3' }\end{array}$ \\
\hline scrambled siRNA & $\begin{array}{l}5^{\prime} \text { - CCUACGCCACCAAUUUCGUdTdT-3' } \\
5^{\prime} \text {-dTdTGGAUGCGGUGGUAAAGCA-3' }\end{array}$ \\
\hline Cy5.5-labeled siRNA & $\begin{array}{l}5^{\prime} \text { - Cy5.5-CCUACGCCACCAAUUUCGUdTdT-3' } \\
5^{\prime} \text {-dTdTGGAUGCGGUGGUUAAAGCA-3' }\end{array}$ \\
\hline PD-1 forward primer & $5^{\prime}$-GACCAGCTTTTGAAGGGAAA-3' \\
\hline PD-1 reverse primer & $5^{\prime}$-GTCCCTAGAAGTGCCCAACA-3' \\
\hline PD-L1 forward primer & $5^{\prime}$ - GACCAGCTTTTGAAGGGAAA-3' \\
\hline PD-L1 reverse primer & $5^{\prime}$ - TGGTTGATTTTGCGGTATGG-3' \\
\hline$\beta$-actin reverse primer & $5^{\prime}$ '-AGAGGGAAATCGTGCGTGAC-3' \\
\hline
\end{tabular}

Table S1. Oligonucleotide sequences of siRNAs and DNA primers. 